\title{
Isolated Perfused Rat Livers to Quantify the Pharmacokinetics and Concentrations of Gd-BOPTA
}

\author{
Catherine M. Pastor $\mathbb{i D}^{1,2}$ \\ ${ }^{1}$ Department of Radiology, Hôpitaux Universitaires de Genève, Geneva, Switzerland \\ ${ }^{2}$ Laboratory of Imaging Biomarkers, Centre of Research on Inflammation, UMR 1149 Inserm, University Paris Diderot, \\ Paris, France \\ Correspondence should be addressed to Catherine M. Pastor; catherine.pastor@unige.ch
}

Received 10 January 2018; Accepted 17 May 2018; Published 11 July 2018

Academic Editor: Anne Roivainen

Copyright (C) 2018 Catherine M. Pastor. This is an open access article distributed under the Creative Commons Attribution License, which permits unrestricted use, distribution, and reproduction in any medium, provided the original work is properly cited.

\begin{abstract}
With recent advances in liver imaging, the estimation of liver concentrations is now possible following the injection of hepatobiliary contrast agents and radiotracers. However, how these images are generated remains partially unknown. Most experiments that would be helpful to increase this understanding cannot be performed in vivo. For these reasons, we investigated the liver distribution of the magnetic resonance (MR) contrast agent gadobenate dimeglumine (Gd-BOPTA, MultiHance ${ }^{\circledR}$, Bracco Imaging) in isolated perfused rat livers (IPRLs). In IPRL, we developed a new set up that quantifies simultaneously the Gd-BOPTA compartment concentrations and the transfer rates between these compartments. Concentrations were measured either by MR signal intensity or by count rates when the contrast agent was labelled by $\left[{ }^{153} \mathrm{Gd}\right]$. With this experimental model, we show how the Gd-BOPTA hepatocyte concentrations are modified by temperature and liver flow rates. We define new pharmacokinetic parameters to quantify the canalicular transport of Gd-BOPTA. Finally, we present how transfer rates generate Gd-BOPTA concentrations in rat liver compartments. These findings better explain how liver imaging with hepatobiliary radiotracers and contrast agents is generated and improve the image interpretation by clinicians.
\end{abstract}

\section{Introduction}

In the past, great achievements were made by analysing the drug plasma concentrations to understand their body distribution. At that time, the estimation of liver concentrations was not available. Therefore, when conducting pharmacokinetic studies, it was assumed that hepatocyte concentrations approximate plasma concentrations, the drug equilibration across the sinusoidal membrane being obtained by passive diffusion. With the discovery of hepatocyte transporters that modify the transport rates across the hepatocyte membranes, this assumption is no longer valid. The activity of sinusoidal transporters can be much higher than passive diffusion, increasing the hepatocyte concentrations over the plasma concentrations. Moreover, the drug concentrations generated by the hepatocyte uptake clearances are simultaneously modified by efflux clearances from hepatocytes into bile canaliculi and back into sinusoids. Thus, depending on the relative hepatocyte influx and efflux clearances, drug hepatocyte concentrations can exceed, equal, or be lower than plasma concentrations. Disconnection between the hepatocyte and plasma concentrations is even more unpredictable when the expression and/or function of membrane transporters are altered.

With recent advances in liver imaging, the estimation of liver concentrations is now possible following the injection of hepatobiliary contrast agents and radiotracers. To study more specifically the activity of hepatocyte transporters in vivo, radiotracers for positron emission tomography (PET) and single-photon emission computed tomography (SPECT) imaging and gadolinium complexes for magnetic resonance imaging (MRI) can be injected before the image acquisition [1-4]. However, how these images are generated remains partially unknown. Moreover, most experiments that would 
be helpful to increase this understanding cannot be performed in vivo. For these reasons, we investigated the liver distribution of the magnetic resonance (MR) contrast agent gadobenate dimeglumine (Gd-BOPTA, MultiHance ${ }^{\circledR}$, Bracco Imaging) in isolated perfused rat livers (IPRLs).

In contrast to cultured cells, IPRL maintains the liver architecture and conserves the lobular distribution of drug transporters and metabolising enzymes. All other cells that may interfere with hepatocyte functions are present, and the bile excretion is preserved. Thus, IPRL simulates in vivo conditions, while avoiding interferences with other organs. In pharmacology, IPRLs are mainly used to measure pharmacokinetic parameters of injected drug, such as the liver extraction ratios, the synthesis of metabolites by hepatocyte enzymes, and the bile excretion of drugs and their metabolites. Several reviews described the various ways to perfuse rodent livers and the numerous investigations that can be performed with this experimental model [5-7].

In IPRL, we quantify simultaneously the Gd-BOPTA concentrations in each liver compartment and the transfer rates between these compartments. The concentrations were measured either by MR signal intensity or by count rates when the contrast agent was labelled by $\left[{ }^{153} \mathrm{Gd}\right]$. Although numerous research teams have been using IPRL to measure clearances from sinusoids to bile canaliculi as well as from hepatocytes back to sinusoids, we are the first group that combines these kinetic parameters with the quantification of concentrations. In this review, we show how Gd-BOPTA hepatocyte concentrations are modified by liver temperature and liver flow rates. We define new pharmacokinetic parameters to quantify the multiple resistance-associated protein 2 (Mrp2) function, and present how transfer rates generate the Gd-BOPTA concentrations in rat liver compartments. These findings better explain how liver imaging with hepatobiliary radiotracers and contrast agents is generated and improve the image interpretation by clinicians.

\section{Methods}

2.1. How We Perfuse Rat Livers? Following the anaesthesia of male Sprague Dawley rats, we cannulate the portal vein, the hepatic artery being too small to be perfused. A KrebsHenseleit bicarbonate (KHB) solution is pumped without delay through the catheter, while the solution is discarded by a vena cava transection following its liver distribution. Liver flow rate is slowly increased over one $\mathrm{min}$ up to $30 \mathrm{ml} / \mathrm{min}$. In a second step, the chest is opened and a second cannula is inserted into the right atrium to collect solutions flowing from the hepatic veins. Finally, the abdominal inferior vena cava is ligated allowing all solutions perfused by the portal vein to be eliminated by the hepatic veins. The entire perfusion system includes a reservoir, a pump, a heating circulator, a bubble trap, a filter, and an oxygenator. Solutions of perfusion are equilibrated with a mixture of $95 \% \mathrm{O}_{2}-5 \% \mathrm{CO}_{2}$, allowing normal liver functions during $120 \mathrm{~min}$. Livers are perfused with the KHB buffer \pm contrast agents or radiotracers using a non-recirculating system. Thus, livers are perfused with newly prepared solutions throughout the protocol. In each experiment, the common bile duct is cannulated with a thin

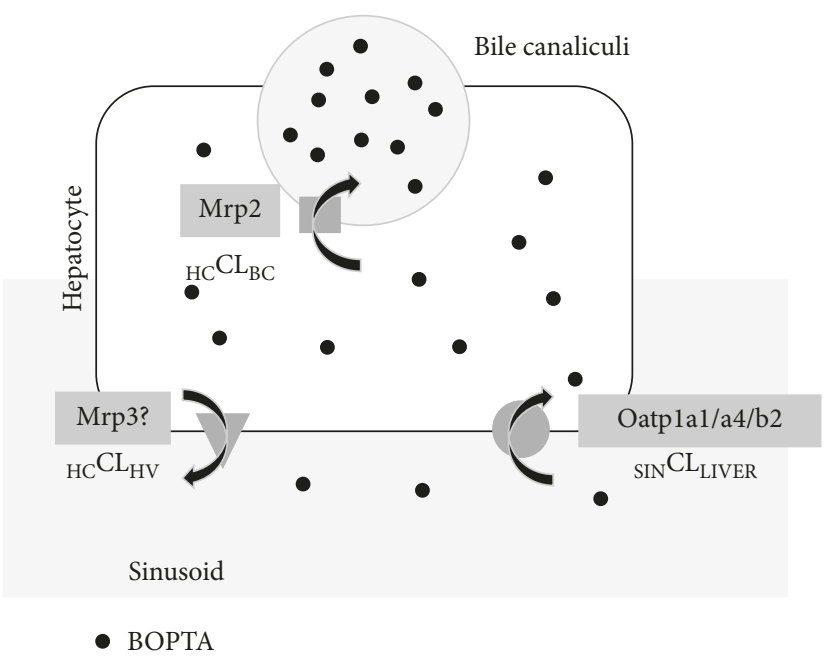

FIgURE 1: Gd-BOPTA transport across hepatocyte membranes. The MRI contrast agent distributes into sinusoids and interstitium before entry into rat hepatocytes across the organic anion transporting polypeptides (Oatp1a1/a4/b2). Once inside hepatocytes, Gd-BOPTA exits into bile canaliculi (BC) across the multiple resistance-associated protein 2 (Mrp2) or back into sinusoids across the sinusoidal membrane. Gd-BOPTA liver concentrations are estimated by liver MRI or quantified with a gamma counter when the molecule is labelled with ${ }^{153} \mathrm{Gd}$. Sinusoidal clearance to liver ( ${ }_{\text {SIN }} \mathrm{CL}_{\mathrm{LIVER}}, \mathrm{ml} / \mathrm{min}$ ) is $\left[\left(C_{\mathrm{PV}}-C_{\mathrm{HV}}\right) \times 30\right] / C_{\mathrm{PV}}$, where $C_{\mathrm{PV}}$ is the concentration $(\mu \mathrm{M})$ in portal vein and $30 \mathrm{ml} / \mathrm{min}$ is the constant liver flow rates. The clearance from hepatocytes to bile canaliculi $\left(\mathrm{HC}_{\mathrm{C}} \mathrm{CL}_{\mathrm{BC}}, \mathrm{ml} / \mathrm{min}\right)$ is ( $C_{\mathrm{BC}} \times$ bile flow rates) $/ C_{\mathrm{HC}}$, where $C_{\mathrm{BC}}$ is the Gd-BOPTA concentration in bile canaliculi and $C_{\mathrm{HC}}$ is the Gd-BOPTA concentration in hepatocytes. The clearance from hepatocytes to interstitium ( $\left.\mathrm{HC}_{\mathrm{CN}}, \mathrm{ml} / \mathrm{min}\right)$ is $\left(C_{\mathrm{HV}} \times 30\right) / C_{\mathrm{HC}}$, where $C_{\mathrm{HV}}$ is the Gd-BOPTA concentration in the hepatic veins. The Gd-BOPTA concentrations (illustrated by the number of circles) increase from sinusoids, to hepatocytes, and to bile canaliculi.

catheter to collect bile samples and measure the bile flow rates. Perfusate samples are also collected from the hepatic veins.

2.2. Properties of Gd-DTPA and Gd-BOPTA. Gadopentetate dimeglumine (Magnevist ${ }^{\circledR}$, Bayer Pharma, Gd-DTPA) is a MRI contrast agent that diffuses exclusively into the extracellular space of the liver [8]. The hepatocyte uptake and bile excretion are negligible, and its overall body excretion occurs by glomerular filtration. In contrast, following an intravenous injection, Gd-BOPTA distributes into the extracellular space and enters into hepatocytes [9]. This contrast agent is highly soluble in water and exhibits a weak plasma protein binding $(<5 \%)$ that is efficient enough to increase the MR signal intensity in plasma and tissues [10]. Gd-BOPTA is not metabolised in hepatocytes. In Xenopus laevis oocytes, we show that the rat sinusoidal transporters of Gd-BOPTA are the organic anion transporting polypeptides la1 (Oatp1a1), Oatp1a4, and Oatp1b2 [11] (Figure 1). Following the study of de Haën et al. [12], we confirmed that Gd-BOPTA is transported into bile canaliculi through the multiple resistance-associated protein 2 (Mrp2) [11]. In liver perfused from rats lacking Mrp2, no Gd-BOPTA is present in bile samples. 


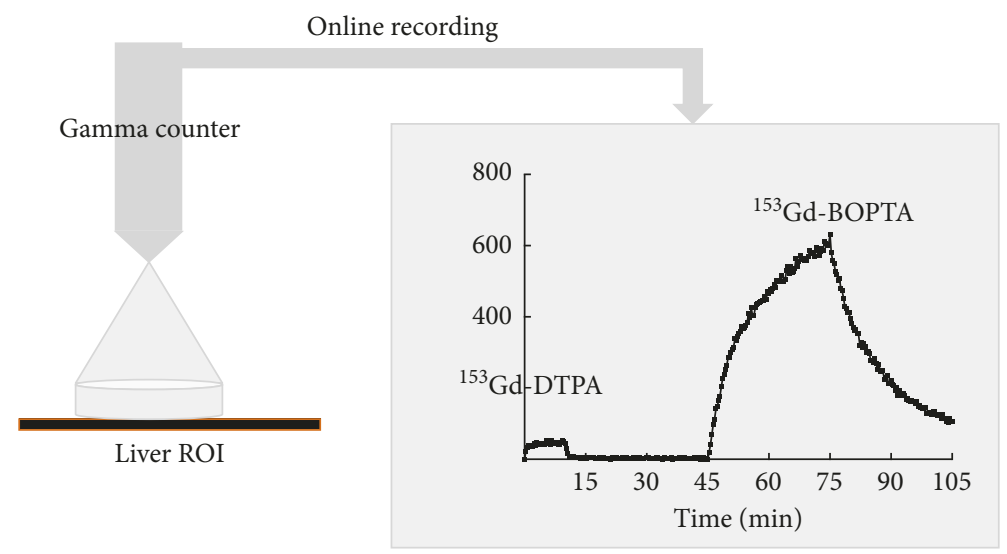

(a)
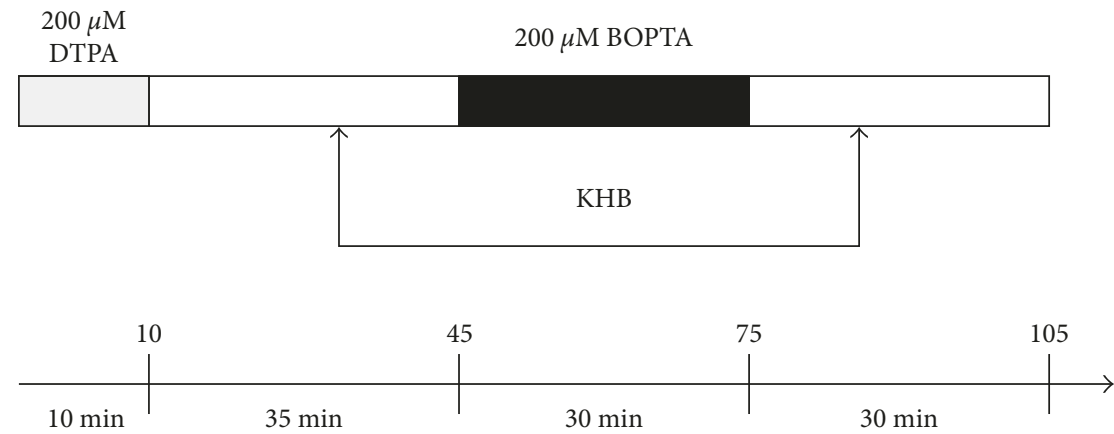

(b)

Figure 2: Protocol of the isolated perfused rat livers. (a) A gamma counter detects online the count rates coming from a region of interest (ROI) delineated within a rat liver lobe. The system avoids traumatic collection of small biopsies over time. (b) We standardise the perfusion protocol to compare results in various experimental conditions. Rat livers are perfused successively with Gd-DTPA that distributes into the extracellular space and Gd-BOPTA that enters into hepatocytes after extracellular distribution. Between drug perfusions, Krebs-Henseleit bicarbonate (KHB) solutions are perfused (rinse periods).

\subsection{Perfusion of Contrast Agents and Radiotracers. Over the} years, the perfusion protocol has been standardised to compare the results in various experimental conditions. Gd-DTPA is perfused during $10 \mathrm{~min}$ at the beginning of the protocol to quantify the liver concentrations generated by its distribution into the extracellular space (Figure 2). Then, a KHB solution rinses the liver from Gd-DTPA. Gd-BOPTA is perfused during $30 \mathrm{~min}$ to quantify its accumulation into the extracellular space and hepatocytes. Livers are finally rinsed with KHB solution during $30 \mathrm{~min}$. Thus, two periods are distinguished: (1) the Gd-BOPTA perfusion period that evidences how the contrast accumulates in the liver compartments, and (2) the Gd-BOPTA rinse period that investigates how the contrast leaves hepatocytes into bile canaliculi and back into sinusoids. To quantify both contrast agents with the gamma counter, we add ${ }^{153} \mathrm{GdCl}_{3}$ to $0.5 \mu \mathrm{M}$ Gd-BOPTA or Gd-DTPA solutions $(1 \mathrm{MBq} / \mathrm{ml})$. ${ }^{153} \mathrm{Gd}-\mathrm{DTPA}$ and ${ }^{153} \mathrm{Gd}$-BOPTA are diluted in KHB solution to obtain $200 \mu \mathrm{M}$ solutions. Unlabelled Gd-DTPA and Gd-BOPTA are used when imaging is performed in the MRI room. In these last experiments, $500 \mu \mathrm{M}$ Gd-DTPA and Gd-BOPTA are perfused. and Hepatocytes. In livers perfused with unlabelled Gd-DTPA and Gd-BOPTA, the only available parameter is the signal intensity enhancement, similarly to clinical studies. Transverse images are acquired in a $1.5 \mathrm{~T}$ MR system with a fast gradient-echo T1-weighted MR sequence. A region of interest is drawn on the short-axis view of the liver, excluding all large vessels. The region of interest remains constant during the entire experiment. However, the MRI room is not a userfriendly environment and sample collection is limited. To overcome this disadvantage, we set up the IPRL in a laboratory dedicated to experiments with radioactivity. To quantify the liver concentrations of ${ }^{153} \mathrm{Gd}-\mathrm{DTPA}$ and ${ }^{153} \mathrm{Gd}$-BOPTA, a gamma counter that measures radioactivity online is placed $1 \mathrm{~cm}$ above rat liver (Figure 2). The counter measures the radioactivity in a region of interest inside the same liver lobe in each experiment. To transform the radioactivity count rates measured by the counter into ${ }^{153} \mathrm{Gd}$-BOPTA concentrations, the radioactivity contained in the liver at the end of each experiment is measured and related to the last count rates detected by the counter. The concentrations in the common bile duct and the hepatic veins are measured every $5 \mathrm{~min}$ in a gamma counter. Concentrations are expressed in $\mu \mathrm{M}$ in the great vessels, the bile duct, and the livers.

The gamma counter placed over the liver lobe quantifies simultaneously count rates originating from sinusoids, interstitium, bile canaliculi, and hepatocytes. To estimate the 
${ }^{153} \mathrm{Gd}$-BOPTA concentrations in bile canaliculi, we multiply the bile concentrations measured in the common bile duct by $0.43 \%$, which corresponds to the relative volume of bile canaliculi according to Blouin et al. [13]. Knowing GdBOPTA concentrations in bile canaliculi and extracellular compartment (estimated by Gd-DTPA concentrations), we subtract both values from the Gd-BOPTA total liver concentrations to obtain Gd-BOPTA concentrations in the volume of hepatocytes within the region of interest. Finally, because Gd-BOPTA concentrations originate from a $78 \%$ volume of hepatocytes, we must increase the values to $100 \%$ to obtain the true hepatocyte concentrations [13].

\subsection{Transfer Rates across Hepatocyte Membranes. In IPRL,} we can easily measure the transfer rates across hepatocyte transporters (Figure 1) [14]. Elimination from sinusoids to livers (sINELIMR LIVER, $\mathrm{nmol} / \mathrm{min}$ ) is measured by $\left(C_{\mathrm{PV}}-C_{\mathrm{HV}}\right) \times 30$ (constant portal blood flow, $\mathrm{ml} / \mathrm{min}$ ), and sinusoidal clearance to liver ( $\operatorname{SIN}_{\mathrm{CI}} \mathrm{LL}_{\mathrm{LIVR}}, \mathrm{ml} / \mathrm{min}$ ) is ${ }_{{ }_{\text {IIN }}} \mathrm{ELIMR}_{\mathrm{LIVER}} / C_{\mathrm{PV}}$, where $C_{\mathrm{PV}}$ is the Gd-BOPTA concentration perfused via the portal vein and $C_{\mathrm{HV}}$ is the Gd-BOPTA concentrations measured in the hepatic veins. The transfer rates from hepatocytes to bile canaliculi or interstitium are also measured. ${ }_{\mathrm{HC}} \operatorname{ELIMR}_{\mathrm{BC}}(\mathrm{nmol} / \mathrm{min})$ is $C_{\mathrm{BC}} \times$ bile flow rate, where $C_{\mathrm{BC}}$ is the Gd-BOPTA concentration in bile canaliculi. The ${ }_{\mathrm{HC}} \mathrm{ELIMR}_{\mathrm{INT}}(\mathrm{nmol} / \mathrm{min})$ is $C_{\mathrm{HV}} \times$ liver flow rate, where $C_{\mathrm{HV}}$ is the concentrations in hepatic veins. The ${ }_{\mathrm{HC}} \mathrm{CL}_{\mathrm{BC}}\left(\mathrm{HC}_{\mathrm{CLIMR}} \mathrm{BC}_{\mathrm{BC}} / C_{\mathrm{HC}}\right)$ and ${ }_{\mathrm{HC}} \mathrm{CL}_{\mathrm{HV}}$ $\left({ }_{\mathrm{HC}} \mathrm{ELIMR}_{\mathrm{HV}} / C_{\mathrm{HC}}\right)$ are also calculated, where $C_{\mathrm{HC}}$ is the Gd-BOPTA concentration in hepatocytes.

2.6. Canalicular Transport Activity of Mrp2. To assess Mrp2 function, we calculate the Gd-BOPTA gradients between the bile and hepatocyte concentrations over time (Figure 5(c)). Another way to illustrate Gd-BOPTA canalicular transport through Mrp2 is to plot its hepatocyte concentrations ( $x$-axis) and its bile concentrations ( $y$-axis) (Figure 5(d)) [15]. To analyse these nonlinear regression curves, we fit the experimental values with the equation $A+B X+C X^{2}$, where $A$ is the intercept on the $y$-axis, $B$ is the slope of regression, and $C$ is the plateau at maximal concentrations. Finally, to understand whether the canalicular fluid transport, the Gd-BOPTA bile concentrations, or both interfere with the Gd-BOPTA excretion rates into bile, we plot the bile excretion rates ( $x$-axis) with the bile flow rates ( $y$-axis), as previously published (Figure 5(b)) [16]. For these investigations, we perfused rat livers with the single-photon emission computed tomography radiotracers ${ }^{99 \mathrm{~m}}$ Tc-DTPA (TechneScan DTPA ${ }^{\circledR}$, b.e.imaging, Schwyz, Switzerland) and Mebrofenin $\left({ }^{99 \mathrm{~m}} \mathrm{Tc}-\mathrm{MEB}\right.$, Choletec $^{\circledR}$, Bracco Imaging) [17-19]. MEB is transported from sinusoids into bile canaliculi by the same transporters as Gd-BOPTA, but its extraction ratio is much higher (94\%) than that of Gd-BOPTA (8\%). DTPA is labelled with ${ }^{99 \mathrm{~m}} \mathrm{Tc}$ (25 mg, $7 \mathrm{MBq}$ ), while MEB is labelled with ${ }^{99 \mathrm{~m}} \mathrm{Tc}(40 \mathrm{mg}$, $11 \mathrm{MBq}$ ). DTPA and MEB are diluted to obtain $64 \mu \mathrm{M}$ concentrations. Thus, we can compare the Mrp2 function of one contrast agent and one radiotracer that display the same hepatocyte transport but different pharmacokinetics.

\section{Experimental Results}

3.1. Gd-BOPTA Concentrations in Normal Livers. In the initial study, we measure the liver signal intensity during the perfusion of Gd-DTPA and Gd-BOPTA (Figure 3) [20]. Liver enhancement with Gd-DTPA rapidly reaches a steady state, while liver enhancement increases over the 30 min perfusion period (Figure 3(a)). During the rinse period, the signal intensity does not return to baseline values as observed with Gd-DTPA. Pharmacokinetic modelling estimates that the half-lives of Gd-DTPA entry and exit are identical (1.3 \pm $0.9 \mathrm{~min}$ ) and shorter than those observed with Gd-BOPTA $(4.8 \pm 0.3 \mathrm{~min}$ for entry and $17.5 \pm 2.8 \mathrm{~min}$ for exit). Interestingly, the coperfusion of the dye bromosulfophthalein (BSP) with Gd-BOPTA prevents the contrast agent entry into hepatocytes and the mean half-lives are similar to those measured with Gd-DTPA (Figures 3(a)-3(c)). This BSP inhibition of hepatocyte Gd-BOPTA uptake is the first drugdrug interaction evidenced by liver imaging. Acute bile duct ligation at the time of surgery does not interfere with GdBOPTA uptake but slows down the decreased signal intensity during the rinse period (Figure 3(a)). These MR results are confirmed when we measure Gd concentrations by inductively coupled plasma atomic emission spectrometry in liver biopsies (Figure 3(b)). In the portal and hepatic veins, the concentrations remain close to $500 \mu \mathrm{M}$ at steady state.

These initial experiments demonstrate that contrast agents can be successively imaged in IPRL. The Gd-DTPA perfusion estimates the signal intensity increase associated with the extracellular volume distribution. According to its fast and steady distribution, additional signal intensity observed during Gd-BOPTA perfusion relates to the hepatocyte entry and the transfer into bile canaliculi. Because contrast agents are perfused with a $\mathrm{KHB}$ containing no protein, protein binding does not alter the Gd-BOPTA entry. None of the contrast agents modifies the portal pressure or the hepatic $\mathrm{O}_{2}$ consumption [20].

3.2. Gd-BOPTA Concentrations in Livers with Chronic Biliary Cirrhosis. We then investigate the signal intensity enhancement during Gd-BOPTA perfusion in livers isolated from rats that had a bile duct ligation (BDL) 15, 30, and 60 days before the liver isolation [21]. BDL induces a severe hepatic injury that increases over time with a downregulation of the Gd-BOPTA transporter expression. The extracellular space (assessed by Gd-DTPA imaging) significantly increases with the disease severity. The Gd-BOPTA-induced signal intensity enhancements are similar in control rats, BDL-15 rats, and BDL-30 rats, but decrease significantly in severe cirrhosis (BDL-60 rats). Thus, Gd-BOPTA-induced signal intensity enhancements cannot be related to the transporter expression, emphasising the need for the simultaneous quantification of hepatocyte concentrations and transfer rates across membranes.

3.3. Role of Liver Temperature on Gd-BOPTA Liver Concentrations. In these experiments, normal livers are perfused with ${ }^{153} \mathrm{Gd}$-DTPA $(200 \mu \mathrm{M})$ and ${ }^{153} \mathrm{Gd}$-BOPTA 


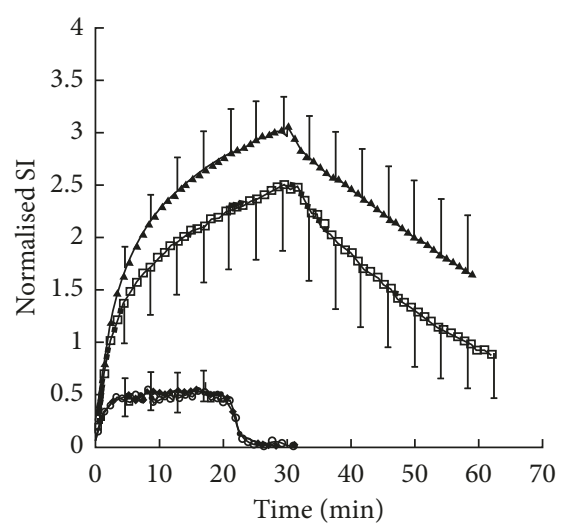

(a)
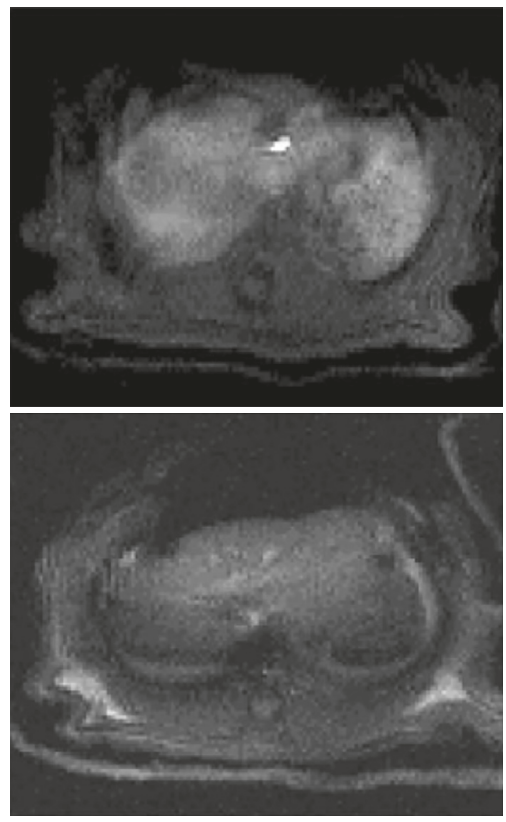

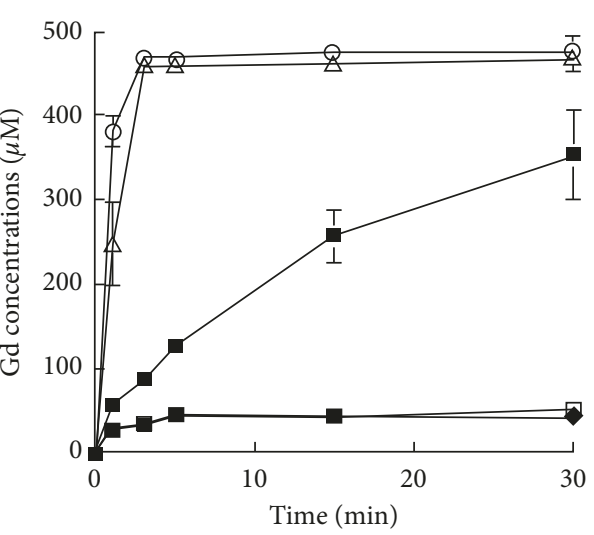

(b)
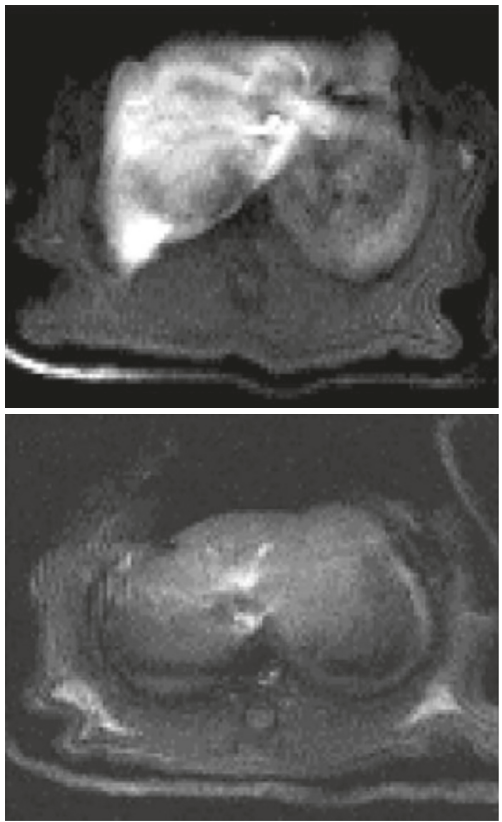

(c)

Figure 3: Gd-BOPTA distribution in rat livers. (a) The livers are perfused with $500 \mu \mathrm{M}$ Gd-DTPA $(\bullet, n=4)$ ), $500 \mu \mathrm{M} \mathrm{Gd-BOPTA}(\square, n=4)$, $500 \mu \mathrm{M}$ Gd-BOPTA and $500 \mu \mathrm{M}$ bromosulfophthalein (BSP, $\bigcirc, n=4$ ). An additional group of rats with acute bile duct ligation is perfused with $500 \mu \mathrm{M}$ Gd-BOPTA $(\boldsymbol{\Lambda}, n=2)$. BSP perfusion prevented Gd-BOPTA uptake. (b) Gadolinium concentrations in portal veins $(\bigcirc)$, hepatic veins $(\triangle)$, and hepatic tissues $(\boldsymbol{\square})$ during Gd-BOPTA perfusion. Additional livers are perfused with Gd-DTPA $(\bullet)$ and Gd-BOPTA + BSP ( $\square$ ). Three liver biopsies are collected at each time point. (c) Representative MR images of a liver perfused with Gd-DTPA and GdBOPTA (upper panels) or DTPA and Gd-BOPTA + BSP (lower panels). Adapted from [20] with permission.

$(200 \mu \mathrm{M})$ at $12,25,30,36$, and $38^{\circ} \mathrm{C}$ to collect samples in the hepatic veins over time (Figure 4(b)) [22]. At the end of the perfusion, liver biopsies are imaged by MRI. The liver ${ }^{153} \mathrm{Gd}-$ DTPA and ${ }^{153} \mathrm{Gd}$-BOPTA concentrations are also measured with a gamma counter (Figure 4(a)). A low amount of GdBOPTA taken up by hepatocytes (3\%) explains the high Gd-BOPTA concentrations measured in hepatic veins (Figure 4(b)). Nevertheless, the maximal Gd-BOPTA concentrations $(188 \pm 5 \mu \mathrm{M})$ in hepatic veins are significantly lower than those of Gd-DTPA $(200 \pm 1 \mu \mathrm{M})$. The MR images of liver biopsies show that the Gd-BOPTA accumulation into hepatocytes declines with temperatures (Figure $4(\mathrm{c})$ ). At $12^{\circ} \mathrm{C}, \mathrm{Gd}$-BOPTA does not enter into hepatocytes and behaves as Gd-DTPA. Temperature variation from 36 to $38^{\circ} \mathrm{C}$ significantly increases Gd-BOPTA hepatocyte accumulation, suggesting that these body temperatures may interfere with imaging in patients.

In these preliminary studies, we show that IPRL is an interesting model to quantify liver concentrations of imaging radiotracers and contrast agents and to assess their temperature dependence. By substituting ${ }^{153} \mathrm{Gd}$ in the Gd-BOPTA molecule, we quantify accurately Gd-BOPTA liver concentrations. However, at that time, we collected liver biopsies for this quantification and few data were available. In the following studies, we place a gamma counter over the liver to record online the accumulation of radiotracers in the livers (Figure 2).

3.4. Canalicular Transport of Gd-BOPTA and MEB across $M r p 2$. Bile excretion of compounds is an important liver 


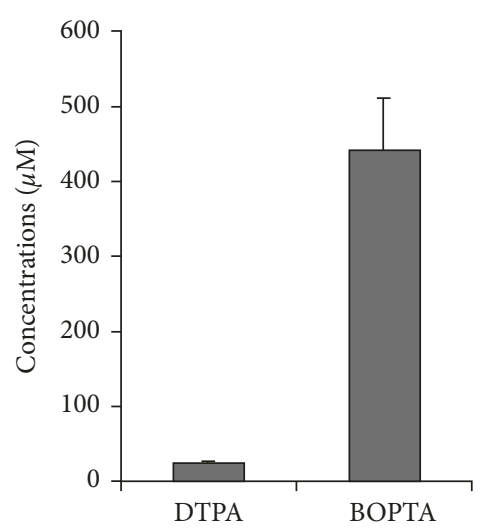

(a)

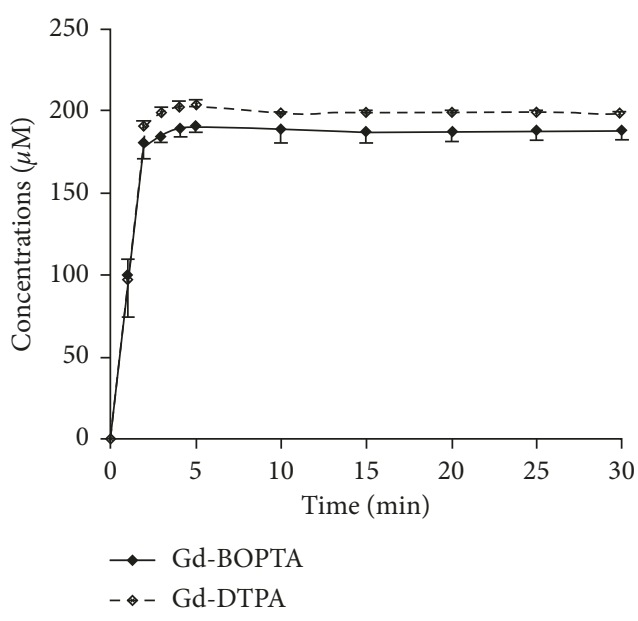

(b)

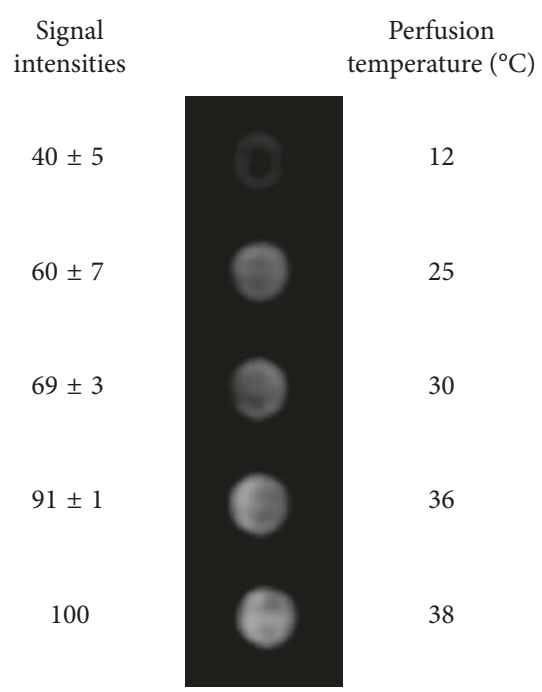

(c)

FIGURE 4: Role of liver temperature on Gd-BOPTA liver concentrations. (a) ${ }^{153}$ Gd-DTPA and ${ }^{153}$ Gd-BOPTA concentrations after a 30 min perfusion $\left(200 \mu \mathrm{M}, 38^{\circ} \mathrm{C}\right)$. (b) Time course of contrast agent concentrations in hepatic veins (mean $\pm \mathrm{SD}, n=3$ ). (c) MR images of tubes containing biopsies of livers perfused during $30 \mathrm{~min}$ with Gd-BOPTA $(200 \mu \mathrm{M})$ at $12,25,30$, 36, and $38^{\circ} \mathrm{C}$. The signal intensity of livers perfused at $38^{\circ} \mathrm{C}$ is $100 \%$. Data are mean $\pm \mathrm{SD}(n=3)$. Adapted from [22] with permission.

function. Primary bile forms at canalicular membrane of adjacent hepatocytes and transports endogenous (bile salts and bilirubin) as well as exogenous (imaging) compounds $[23,24]$. Most compounds that entered hepatocytes cross the canalicular membrane by export pumps. This primary bile is then modified along ductules and ducts by absorptive and secretory processes that take place in the cholangiocyte epithelium [23].

In the IPRL, we investigate two new parameters to assess Mrp2 transport function: the gradients between Gd-BOPTA hepatocyte and bile concentrations over the perfusion period, and a unique parameter named canalicular concentration ratio (CCR) that represents the slope of the nonlinear regression curve between hepatocyte and bile concentrations [15].

\subsubsection{Changes of Bile Flow Rates with Hepatobiliary Com-} pounds. We show that Gd-DTPA perfusion does not modify the bile flow rates (Figure 5(a)). The contrast agent does not enter into hepatocytes and has no bile excretion. Bile flow rates do not change during MEB perfusion, but importantly increase during Gd-BOPTA perfusion. The choleretic effect of Gd-BOPTA is linked to bile concentrations [25]. The bile flow rates return to basal values during the rinse period, according to the gradual decrease of Gd-BOPTA hepatocyte concentrations. To understand whether the canalicular fluid transfer, the bile concentrations, or both interfere with drug excretion rates into bile, we plot the drug bile excretion rates ( $x$-axis) and the bile flow rates ( $y$-axis) as previously published [16] (Figure 5(b)). The regressions clearly show that the bile excretion rates of MEB are driven by the bile concentrations, while the Gd-BOPTA bile excretion rates increase according to both bile concentrations and fluid transfer.
3.4.2. Canalicular Transport Activity across Mrp2. The GdBOPTA and MEB gradients between hepatocyte and bile concentrations over the perfusion period rapidly increase to reach a similar plateau (Figure 5(c)). However, during the rinse period, the gradients have different evolution. By plotting the hepatocyte concentrations ( $x$-axis) and the bile concentrations ( $y$-axis), we find similar regression slopes (CCR) for Gd-BOPTA and MEB (Figure 5(d)). Thus, with these parameters, we can assess the Mrp2 function independently from the cellular uptake of compounds. The shape of these nonlinear regressions confirms that the transport via Mrp2 is saturated at high concentrations (Figure 5(d)). CCR is null in rat livers deficient in Mrp2. CCRs might also be a parameter of Mrp2 inhibition, but we did not yet quantify these data.

\subsection{How Transfer Rates Generate Gd-BOPTA Concentrations} in Rat Liver Compartments. More recently, we measured the true concentrations in hepatocytes and show how transfer rates across sinusoidal and canalicular membranes generate these concentrations without any pharmacokinetic modelling (Figure 1) [14]. Liver parenchyma is divided into sinusoids, interstitium, hepatocytes, and bile canaliculi. In clinical MRI, the averaged signal intensity is quantified in regions of interest, but concentrations from each compartment cannot be estimated. In the common bile duct, the signal intensity estimates true concentrations because the structure has a single compartment. In portal and hepatic veins, they estimate true concentrations once cell volumes are subtracted. In human livers, transfer rates between two liver compartments can only be estimated by pharmacokinetic modelling [26-30]. However, it is important to measure true concentrations inside each compartment, because concentrations across membranes partly regulate transfer rates across them [31]. 


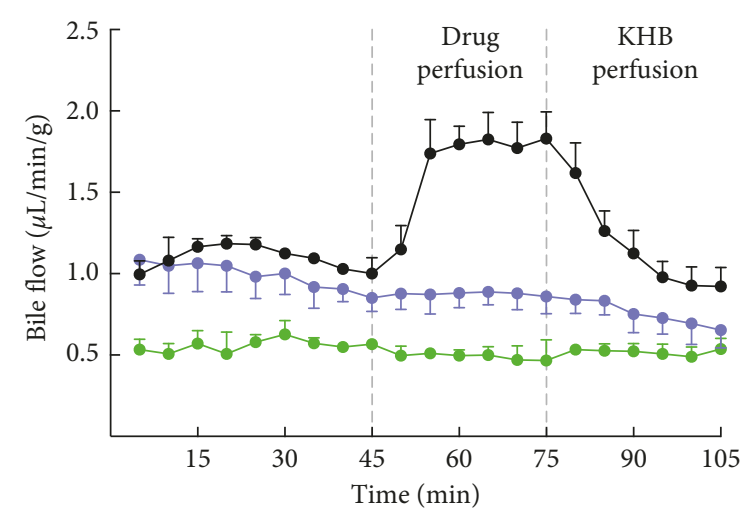

(a)

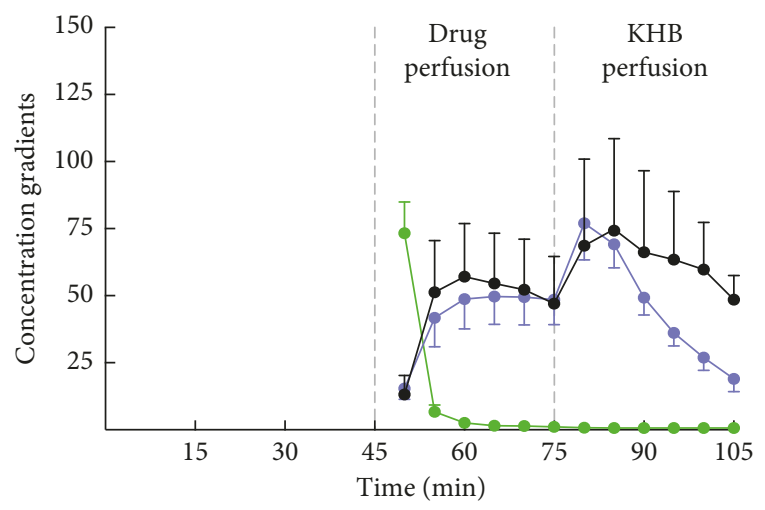

(c)

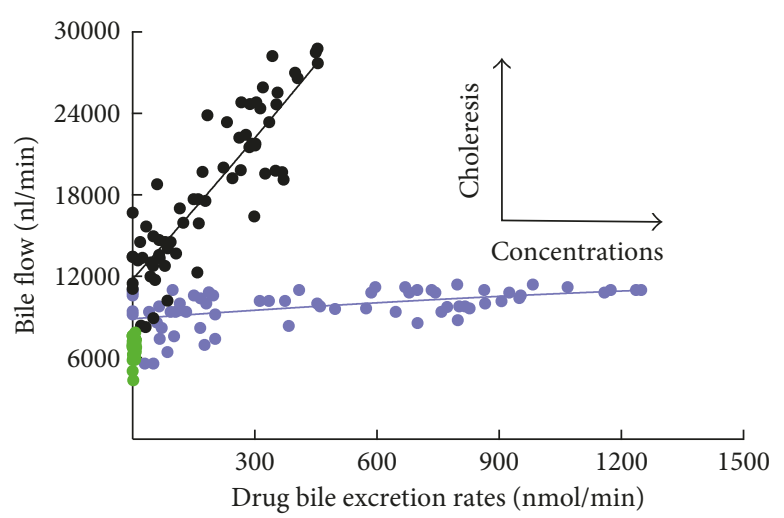

(b)

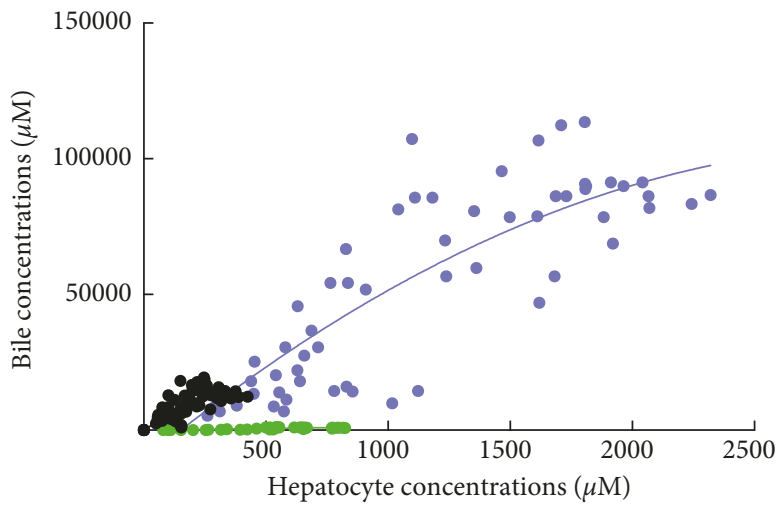

(d)

Figure 5: Canalicular transport across Mrp2. (a) Bile flow rates $(\mu \mathrm{l} / \mathrm{min} / \mathrm{g})$ during the perfusion of Gd-DTPA, Gd-BOPTA, and MEB. (b) Plots between drug bile excretion rates ( $x$-axis, $\mathrm{nmol} / \mathrm{min}$ ) and bile flow rates ( $y$-axis, $\mathrm{nl} / \mathrm{min})$. (c) Concentration gradients between bile canaliculi and hepatocytes over time. (d) Plots between drug hepatocyte concentrations $(x$-axis, $\mu \mathrm{M})$ and drug bile concentrations $(y$-axis, $\mu \mathrm{M})$. Three groups of rat livers are perfused: (1) livers isolated from normal rats and perfused with $200 \mu \mathrm{M}$ Gd-DTPA and $200 \mu \mathrm{M}$ Gd-BOPTA (black circles, $n=5$ ); (2) livers isolated from rats lacking Mrp2 and perfused with $200 \mu \mathrm{M}$ Gd-DTPA and $200 \mu \mathrm{M}$ Gd-BOPTA (green circles, $n=3$ ); and (3) livers isolated from normal rats and perfused with $64 \mu \mathrm{M}$ DTPA and $64 \mu \mathrm{M}$ MEB (blue circles, $n=5$ ). Adapted from [15] with permission.

In normal livers, Gd-BOPTA concentrations increase rapidly over $10 \mathrm{~min}$ (Figure 6(a)) [14]. Thereafter, the increase is slower and the concentrations reach $473 \pm 56 \mu \mathrm{M}$. When Gd-BOPTA perfusion is replaced by the KHB solution, the concentrations steadily decrease. The Gd-BOPTA concentrations in common bile duct (Figure 6(b)) are present $5 \mathrm{~min}$ after the start of perfusion (first sampling), and the maximal true concentrations reach $15700 \pm 3100 \mu \mathrm{M}$ (end of perfusion), a concentration 78 times higher than that perfused in portal veins $(200 \mu \mathrm{M})$. In rats lacking Mrp2, liver concentrations linearly increase during the perfusion period (Figure 6(a)). The concentrations are much higher in livers lacking Mrp2 than in normal livers, while Gd-BOPTA concentrations in the common duct are tiny (Figure 6 (b)). In this group, Gd-BOPTA remains trapped inside hepatocytes until the end of the protocol.

Finally, we can explain how Gd-BOPTA transfer rates generate concentrations in normal hepatocytes (Figures 7(a) and $7(b)$ ). At the beginning of perfusion, the Gd-BOPTA elimination from sinusoids to livers ( $\left.{ }_{\text {IIN }} E_{L I M R}{ }_{\text {LIVER }}\right)$ is much higher than the elimination rates from hepatocytes to bile canaliculi $\left({ }_{\mathrm{HC}} \mathrm{ELIMR}_{\mathrm{BILE}}\right)$. Then, the ${ }_{\text {SIN }}$ ELIMR $_{\text {LIVER }}$ decreases but remains higher than the ${ }_{\mathrm{HC}}$ ELIMR $_{\mathrm{BILE}}$, and the hepatocyte concentrations continue to increase moderately. The absence of ${ }_{\mathrm{HC}}$ ELIMR $_{\mathrm{BILE}}$ clearly explains the high hepatocyte concentrations in livers lacking Mrp2.

3.6. Hepatocyte Transport Activity and Liver Perfusion. To investigate whether changing liver flow rates modifies GdBOPTA hepatocyte concentrations, we isolate livers from normal rats and perfused them at various liver flow rates: $24 \mathrm{ml} / \mathrm{min}(n=3$, Flow 24$), 30 \mathrm{ml} / \mathrm{min}(n=5$, Flow30), or $36 \mathrm{ml} / \mathrm{min}(\mathrm{n}=3$, Flow36) [32]. Of note, the standard liver flow rate is $30 \mathrm{ml} / \mathrm{min}$. The perfused concentration in portal veins is $200 \mu \mathrm{M}$, and changing liver flow rates modifies GdDTPA and Gd-BOPTA delivery rates from $4800 \mathrm{nmol} / \mathrm{min}$ (Flow24), $6000 \mathrm{nmol} / \mathrm{min}$ (Flow30), and $7200 \mathrm{nmol} / \mathrm{min}$ (Flow36). Portal pressures increase with liver flow rates, while bile flow decreases when livers are perfused at the low flow rate. High (Flow36) and low (Flow24) flow rates decrease hepatocyte Gd-BOPTA concentrations in comparison with the standard flow rate $(30 \mathrm{ml} / \mathrm{min}$, Figure $8(\mathrm{~d})$ ). These low hepatocyte concentrations are explained by the 


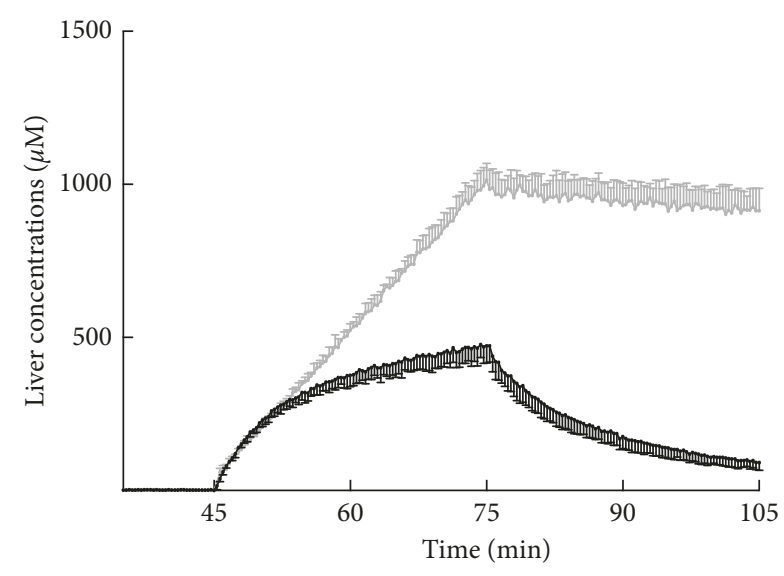

(a)

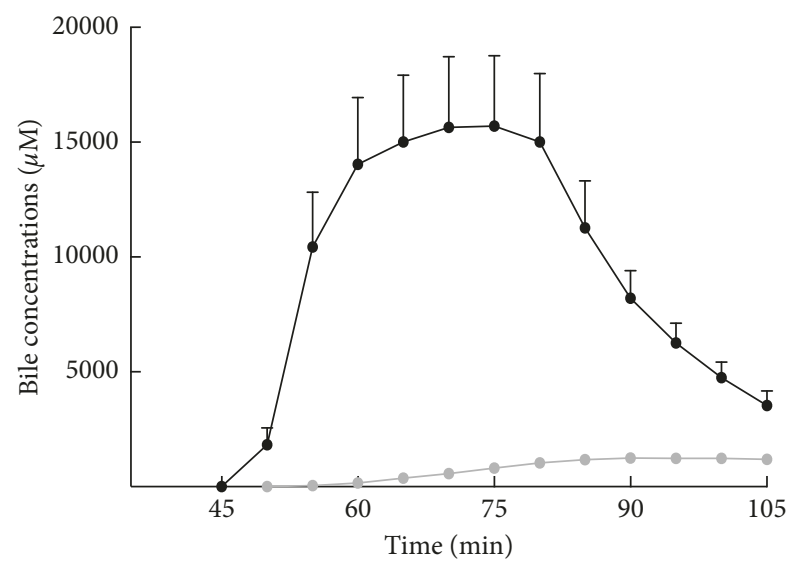

(b)

FIGURE 6: Gd-BOPTA concentrations over time in structures identified at clinical MRI. Values are either liver concentrations assessed by the gamma counter (a) or true concentrations in bile (b). Gd-BOPTA $(200 \mu \mathrm{M})$ is perfused from 45 to $75 \mathrm{~min}$ and replaced by a rinse solution from 75 to $105 \mathrm{~min}$. Two groups of rats are studied: normal rats $(n=5$, black symbols) and rats lacking Mrp2 ( $\mathrm{n}=3$, grey symbols). Adapted from [14] with permission.

low ${ }_{\text {SIN }} \mathrm{CL}_{\text {LIVER }}$ (uptake function) and the high ${ }_{\mathrm{HC}} \mathrm{CL}_{\mathrm{INT}}$ (efflux back), ${ }_{\mathrm{HC}} \mathrm{CL}_{\mathrm{BC}}$ being identical in the 3 groups. Consequently, changing portal flow rates modifies GdBOPTA hepatocyte concentrations, a result important to consider when interpreting liver imaging.

\section{Conclusion}

Our investigations clearly show that MR contrast agents can be imaged in IPRL. With our experimental protocol, the GdDTPA perfusion estimates the signal intensity increase associated with the extracellular volume distribution. According to its fast and steady distribution, additional signals observed during Gd-BOPTA perfusion relate to the hepatocyte entry and the transfer into bile canaliculi. In rats with chronic liver diseases, the Gd-BOPTA-induced enhancements are not related to the modified expression of Gd-BOPTA transporters, emphasising the need for the simultaneous quantification of the hepatocyte concentrations

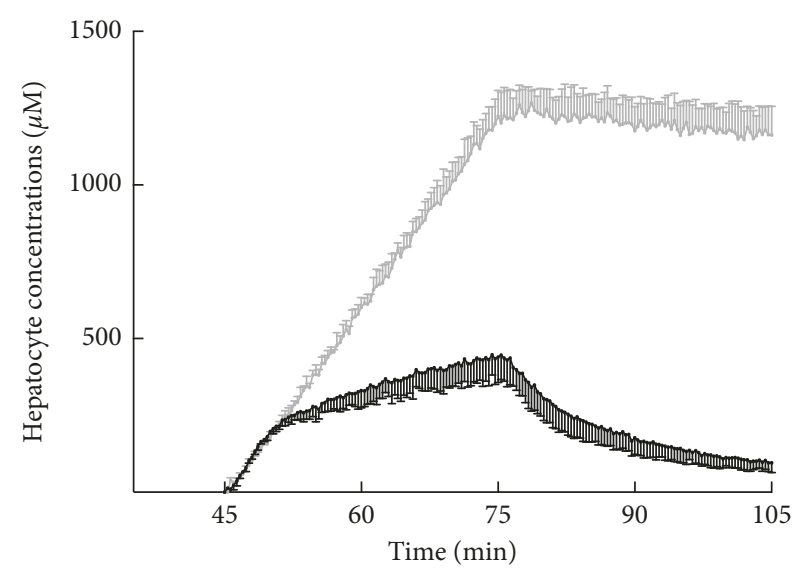

(a)

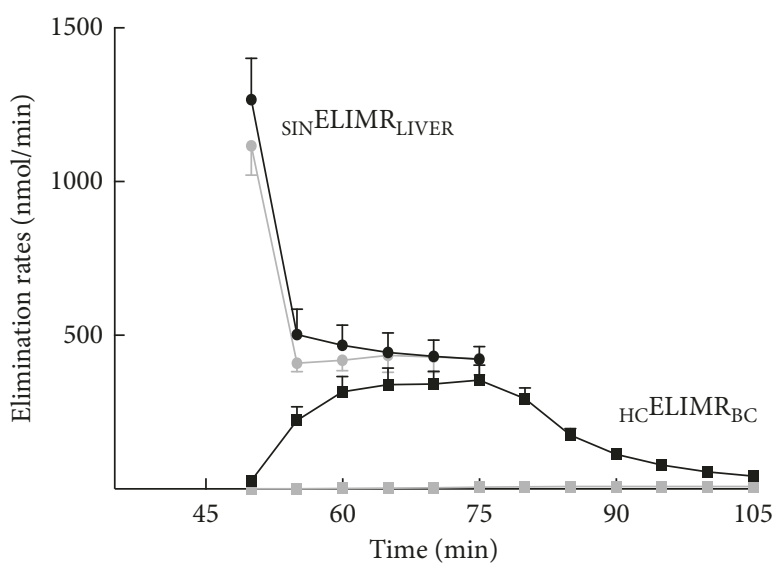

(b)

FIGURE 7: Gd-BOPTA concentrations in hepatocytes over time (a) according to the concomitant Gd-BOPTA elimination rates from sinusoids to liver ( ${ }_{\text {SIN ELIMR }}$ LIVER, $\mathrm{nmol} / \mathrm{min}$ ) and Gd-BOPTA elimination rates from hepatocytes to bile canaliculi ( ${ }_{\mathrm{HC}} \mathrm{ELIMR}_{\mathrm{BC}}$ ) (b). Two groups of rats are studied: normal rats $(n=5$, black symbols) and rats lacking Mrp2 $(n=3$, grey symbols). Livers are perfused with $200 \mu \mathrm{M}$ Gd-BOPTA (45 to $75 \mathrm{~min}$ ) and rinse solution (75 to $105 \mathrm{~min}$ ). Adapted from [14] with permission.

and the transfer rates across cellular membranes in IPRL. In an early study, we assessed the temperature dependence of Gd-BOPTA liver concentrations. We also show that Mrp2 transport function is well characterised by new parameters that are independent of Gd-BOPTA uptake into hepatocytes. More recently, we demonstrate how the transfer rates across sinusoidal and canalicular membranes generate hepatocyte concentrations, and that the liver flow rates alter these concentrations by modifying Gd-BOPTA clearances across the sinusoidal membrane.

IPRL is a convenient model because the experimental conditions are well controlled and simplified. The gamma counter placed over the liver detects the concentrations of imaging compounds, avoiding serial liver biopsies [33]. The delineation of a single region of interest is however a disadvantage in comparison with the $3 \mathrm{D}$ liver imaging. The imaging compounds we use are free to enter into hepatocytes because perfused solutions do not contain proteins. It would be easy to add albumin to the perfused solutions to 


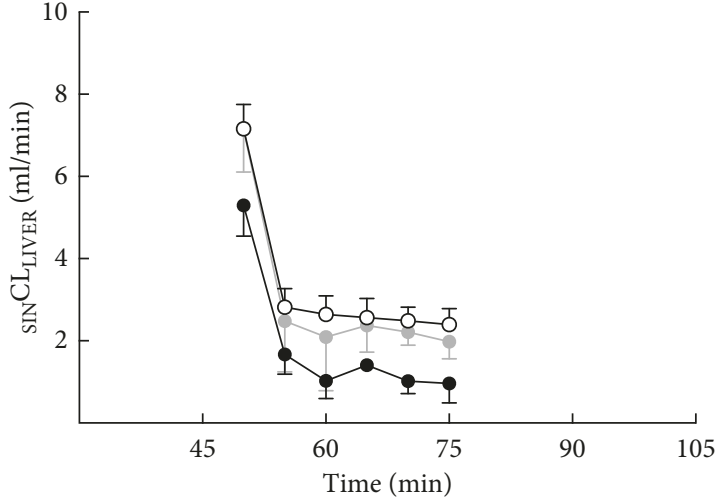

(a)

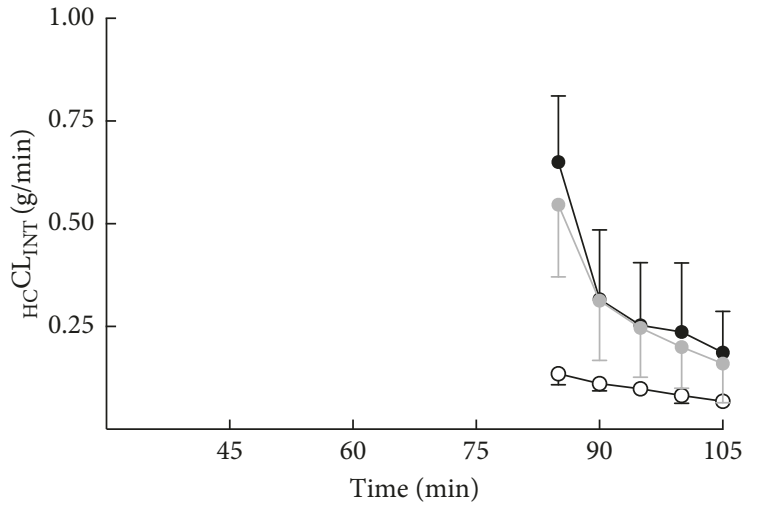

(c)

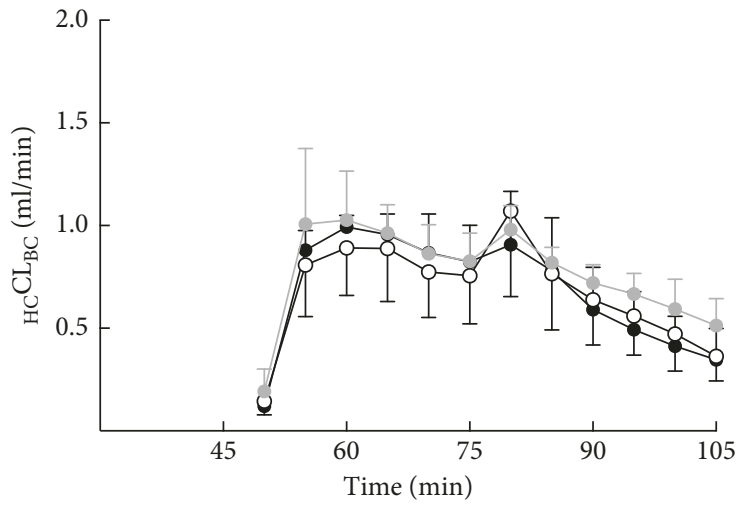

(b)

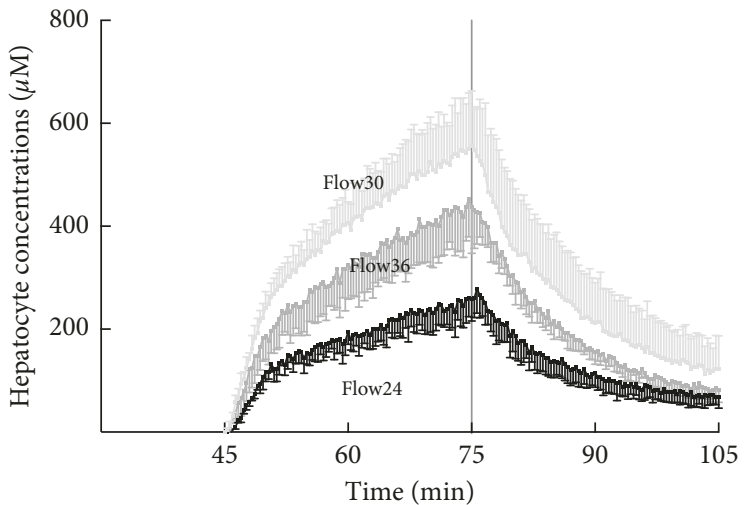

(d)

FIgURE 8: Hepatocyte transport activity and liver perfusion. Gd-BOPTA hepatocyte concentrations (d) over time according to clearances from sinusoids to liver ( ${ }_{S I N} \mathrm{CL}_{\mathrm{LIVER}}, \mathrm{a}$ ), clearances from hepatocytes to bile canaliculi ( $\mathrm{HC}_{\mathrm{CL}} \mathrm{L}_{\mathrm{BC}}, \mathrm{b}$ ), and clearances from hepatocytes back to interstitium ( $\mathrm{HCCL}_{\mathrm{INT}}, \mathrm{c}$ ). Livers are perfused with $200 \mu \mathrm{M}$ Gd-BOPTA (45 to $75 \mathrm{~min}$ ) and Krebs-Henseleit bicarbonate (KHB) solution (75 to $105 \mathrm{~min}$ ) at various flow rates: $24 \mathrm{ml} / \mathrm{min}$ (black circles), $30 \mathrm{ml} / \mathrm{min}$ (white circles), or $36 \mathrm{ml} / \mathrm{min}$ (grey circles). Adapted from [32] with permission.

assess the role of protein binding on the uptake clearances. Livers are perfused only through the portal vein, avoiding the complexity of a dual input entry. A flow rate of $30 \mathrm{ml} / \mathrm{min}$ (or $1 \mathrm{ml} / 10 \mathrm{~g}$ of body weight) is commonly used when livers are perfused with the KHB solution that contains no red blood cells. To investigate the consequences of liver flow rates on Gd-BOPTA hepatocyte concentrations, we decrease the liver flow rate only to $24 \mathrm{ml} / \mathrm{min}$ to avoid liver ischemia. Indeed, we previously published that decreasing liver $\mathrm{O}_{2}$ delivery is associated with an increased $\mathrm{O}_{2}$ extraction ratio to maintain a normal $\mathrm{O}_{2}$ consumption until a threshold where $\mathrm{O}_{2}$ consumption decreases with altered hepatic functions [34]. At $24 \mathrm{ml} / \mathrm{min}$, the bile flow is already altered. Finally, we can measure concentrations in all liver compartments and no pharmacokinetic modelling is necessary to calculate Gd-BOPTA transfer rates across hepatocyte membranes.

The new understanding summarised in the present article was directly translated to human liver imaging in two recent publications. In 2016, we reviewed all the published studies that correlate the hepatobiliary MR imaging and the expression of hepatocyte transporters in human hepatocellular carcinomas [35]. We showed how the understanding of signal intensity in these focal lesions relies on the transport function of the human Mrp2. More recently, we published the pharmacokinetic modelling of liver images to quantify the liver perfusion and hepatocyte transport function of patients with chronic liver diseases [36].

\section{Abbreviations}

Gd- $\quad$ Gadobenate dimeglumine, MultiHance ${ }^{\circledR}$,

BOPTA: Bracco Imaging

CCR: $\quad$ Canalicular concentration ratio

Gd-DTPA: Gadopentetate dimeglumine, Magnevist ${ }^{\circledR}$, Bayer Pharma

${ }^{99 m}$ Tc- $\quad$ TechneScan DTPA ${ }^{\circledR}$, b.e.imaging, Schwyz,

DTPA: $\quad$ Switzerland

IPRL: $\quad$ Isolated perfused rat liver

MEB: $\quad$ Mebrofenin, Choletec ${ }^{\circledR}$, Bracco Imaging

MRI: $\quad$ Magnetic resonance imaging

SPECT: Single-photon emission computed tomography.

\section{Conflicts of Interest}

The author declares that there are no conflicts of interest regarding the publication of this article. 


\section{Authors' Contributions}

Corinne Planchamp, Joëlle Bourquin, and Pierre Bonnaventure contributed greatly to the publications highlighted in the review.

\section{References}

[1] H. Kusuhara, "Imaging in the study of membrane transporters," Clinical Pharmacology and Therapeutics, vol. 94, no. 1, pp. 33-36, 2013.

[2] A. Testa, M. Zanda, C. S. Elmore, and P. Sharma, "PET tracers to study clinically relevant hepatic transporters," Molecular Pharmaceutics, vol. 12, no. 7, pp. 2203-2216, 2015.

[3] O. Langer, "Use of PET imaging to evaluate transportermediated drug-drug interactions," Journal of Clinical Pharmacology, vol. 56, no. 7, pp. S143-S156, 2016.

[4] A. Mann, I. Semenenko, M. Meir, and S. Eyal, "Molecular imaging of membrane transporter activity in cancer: a picture is worth a thousand tubes," AAPS Journal, vol. 17, no. 4, pp. 788-801, 2015.

[5] G. J. Gores, L. J. Kost, and N. F. LaRusso, "The isolated perfused rat liver: conceptual and practical considerations," Hepatology, vol. 6, no. 3, pp. 511-517, 1986.

[6] P. Godoy, N. J. Hewitt, U. Albrecht et al., "Recent advances in $2 \mathrm{D}$ and $3 \mathrm{D}$ in vitro systems using primary hepatocytes, alternative hepatocyte sources and non-parenchymal liver cells and their use in investigating mechanisms of hepatotoxicity, cell signaling and ADME," Archives of Toxicology, vol. 87, pp. 1315-1530, 2013.

[7] A. Ferrigno, P. Richelmi, and M. Vairetti, "Troubleshooting and improving the mouse and rat isolated perfused liver preparation," Journal of Pharmacological and Toxicological Methods, vol. 67, no. 2, pp. 107-114, 2013.

[8] H. J. Weinmann, M. Laniado, and W. Mützel, "Pharmacokinetics of Gd-DTPA/Dimeglumine after intravenous injection into healthy volunteers," Physiological Chemistry and Physics and Medical NMR, vol. 16, pp. 167-172, 1984.

[9] C. de Haën, M. Cabrini, L. Akhnana, D. Ratti, L. Calabi, and L. Gozzini, "Gadobenate dimeglumine 0.5 M solution for injection (MultiHance): pharmaceutical formulation and physicochemical properties of a new magnetic resonance imaging contrast medium," Journal of Computer Assisted Tomography, vol. 23, pp. S161-S168, 1999.

[10] F. M. Cavagna, F. Maggioni, P. M. Castelli et al., "Gadolinium chelates with weak binding to serum proteins. A new class of high-efficiency, general purpose contrast agents for magnetic resonance imaging," Investigative Radiology, vol. 32, no. 12, pp. 780-796, 1997.

[11] C. Planchamp, A. Hadengue, B. Stieger et al., "Function of both sinusoidal and canalicular transporters controls the concentration of organic anions within hepatocytes," Molecular Pharmacology, vol. 71, no. 4, pp. 1089-1097, 2007.

[12] C. de Haën, V. Lorusso, and P. Tirone, "Hepatic transport of gadobenate dimeglumine in TR-rats," Academic Radiology, vol. 3, pp. S452-S454, 1996.

[13] A. Blouin, R. P. Bolender, and E. R. Weibel, "Distribution of organelles and membranes between hepatocytes and nonhepatocytes in the rat liver parenchyma. A stereological study," Journal of Cell Biology, vol. 72, no. 2, pp. 441-455, 1977.

[14] C. M. Pastor, "How transfer rates generate Gd-BOPTA concentrations in rat liver compartments: implications for clinical liver imaging with hepatobiliary contrast agents,"
Contrast Media and Molecular Imaging, vol. 11, no. 4, pp. 291-298, 2016.

[15] P. Bonnaventure and C. M. Pastor, "Quantification of drug transport function across the multiple resistance-associated protein 2 (Mrp2) in rat livers," International Journal of Molecular Sciences, vol. 16, no. 1, pp. 135-147, 2015.

[16] N. Tavoloni, J. S. Reed, and J. L. Boyer, "Hemodynamic effects on determinants of bile secretion in isolated rat liver," American Journal of Physiology-Endocrinology and Metabolism, vol. 234, no. 6, pp. E584-E592, 1978.

[17] B. Swift, W. Yue, and K. L. Brouwer, "Evaluation of (99m) technetium-mebrofenin and $(99 \mathrm{~m})$ technetium-sestamibi as specific probes for hepatic transport protein function in rat and human hepatocytes," Pharmaceutical research, vol. 27, no. 9, pp. 1987-1998, 2010.

[18] W. de Graaf, R. J. Bennink, R. Veteläinen, and T. M. van Gulik, "Nuclear imaging techniques for the assessment of hepatic function in liver surgery and transplantation," Journal of Nuclear Medicine, vol. 51, no. 5, pp. 742-752, 2010.

[19] W. de Graaf, S. Häusler, M. Heger et al., "Transporters involved in the hepatic uptake of $(99 \mathrm{~m}) \mathrm{Tc}-\mathrm{mebrofenin}$ and indocyanine green," Journal of Hepatology, vol. 54, no. 4, pp. 738-745, 2011.

[20] C. M. Pastor, C. Planchamp, S. Pochon et al., "Kinetics of gadobenate dimeglumine in isolated perfused rat liver: MR imaging evaluation," Radiology, vol. 229, no. 1, pp. 119-125, 2003.

[21] C. Planchamp, X. Montet, J.-L. Frossard et al., "Magnetic resonance imaging with hepatospecific contrast agents in cirrhotic rat livers," Investigative Radiology, vol. 40, no. 4, pp. 187-194, 2005.

[22] C. Planchamp, G. J. Beyer, D. O. Slosman, F. Terrier, and C. M. Pastor, "Direct evidence of the temperature dependence of Gd-BOPTA transport in the intact rat liver," Applied Radiation and Isotopes, vol. 62, no. 6, pp. 943-949, 2005.

[23] J. L. Boyer, "Bile formation and secretion," Comprehensive Physiology, vol. 3, pp. 1035-1078, 2013.

[24] A. Esteller, "Physiology of bile secretion," World Journal of Gastroenterology, vol. 14, no. 37, pp. 5641-5649, 2008.

[25] P. Millet, M. Moulin, B. Stieger, Y. Daali, and C. M. Pastor, "How organic anions accumulate in hepatocytes lacking Mrp2: evidence in rat liver," Journal of Pharmacology and Experimental Therapeutics, vol. 336, no. 3, pp. 624-632, 2011.

[26] M. Ingrisch and S. Sourbron, "Tracer-kinetic modeling of dynamic contrast-enhanced MRI and CT: a primer," Journal of Pharmacokinetics and Pharmacodynamics, vol. 40, no. 3, pp. 281-300, 2013.

[27] M. F. Forsgren, O. Dahlqvist Leinhard, N. Dahlström, G. Cedersund, and P. Lundberg, "Physiologically realistic and validated mathematical liver model reveals hepatobiliary transfer rates for Gd-EOB-DTPA using human DCE-MRI data," PLoS One, vol. 9, no. 4, Article ID e95700, 2014.

[28] S. Sourbron, W. H. Sommer, M. F. Reiser, and C. J. Zech, "Combined quantification of liver perfusion and function with dynamic gadoxetic acid-enhanced MR imaging," Radiology, vol. 263, no. 3, pp. 874-883, 2012.

[29] K. Juluru, A. H. Talal, R. K. Yantiss et al., "Diagnostic accuracy of intracellular uptake rates calculated using dynamic GdEOB-DTPA-enhanced MRI for hepatic fibrosis stage," Journal of Magnetic Resonance Imaging, vol. 45, no. 4, pp. 1177-1185, 2017.

[30] H. Nilsson, L. Blomqvist, L. Douglas et al., "Gd-EOB-DTPAenhanced MRI for the assessment of liver function and 
volume in liver cirrhosis," British Journal of Radiology, vol. 86, no. 1026, article 20120653, 2013.

[31] X. Chu, K. Korzekwa, R. Elsby et al., "Intracellular drug concentrations and transporters: measurement, modeling, and implications for the liver," Clinical Pharmacology and Therapeutics, vol. 94, no. 1, pp. 126-141, 2013.

[32] J. L. Daire, B. Leporq, V. Vilgrain, B. E. Van Beers, S. Schmidt, and C. M. Pastor, "Liver perfusion modifies Gd-DTPA and Gd-BOPTA hepatocyte concentrations through transfer clearances across sinusoidal membranes," European Journal of Drug Metabolism and Pharmacokinetics, vol. 42, no. 4, pp. 657-667, 2017.

[33] F. Cusin, L. Fernandes Azevedo, P. Pierre Bonnaventure, Y. Daali, and C. M. Pastor, "Imaging with ICG reflects hepatocyte concentrations and relies on transfer rates across membrane transporters," Basic and Clinical Pharmacology and Toxicology, vol. 120, no. 2, pp. 171-178, 2017.

[34] C. M. Pastor, D. R. Morel, and T. R. Billiar, "Oxygen supply dependence of urea production in the isolated perfused rat liver," American Journal of Respiratory and Critical Care Medicine, vol. 157, no. 3, pp. 796-802, 1998.

[35] V. Vilgrain, B. E. Van Beers, and C. M. Pastor, "Insights into the diagnosis of hepatocellular carcinomas with hepatobiliary MRI," Journal of Hepatology, vol. 64, no. 3, pp. 708-716, 2016.

[36] B. Leporq, J. L. Daire, C. M. Pastor et al., "Quantification of hepatic perfusion and hepatocyte function with dynamic gadoxetic acid-enhanced MRI in patients with chronic liver disease," Clinical Science, vol. 132, no. 7, pp. 813-824, 2018. 


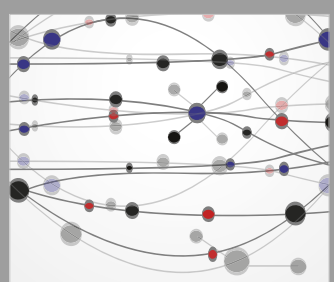

The Scientific World Journal
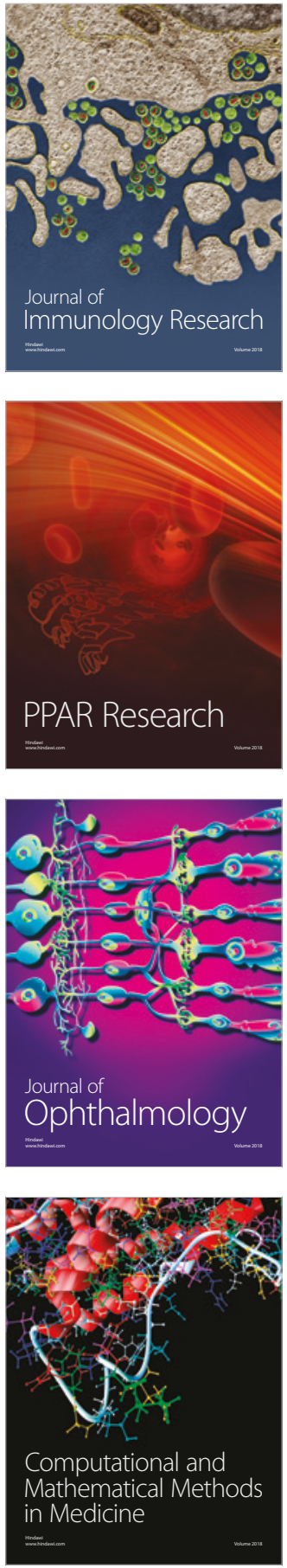

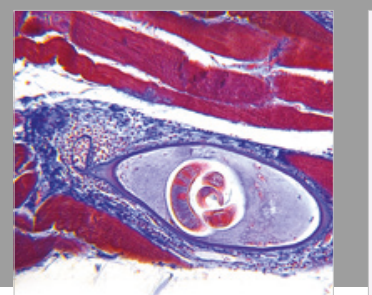

Gastroenterology Research and Practice

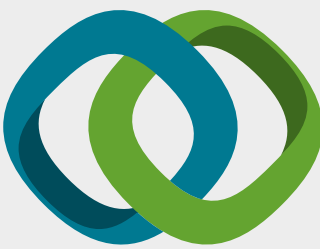

\section{Hindawi}

Submit your manuscripts at

www.hindawi.com
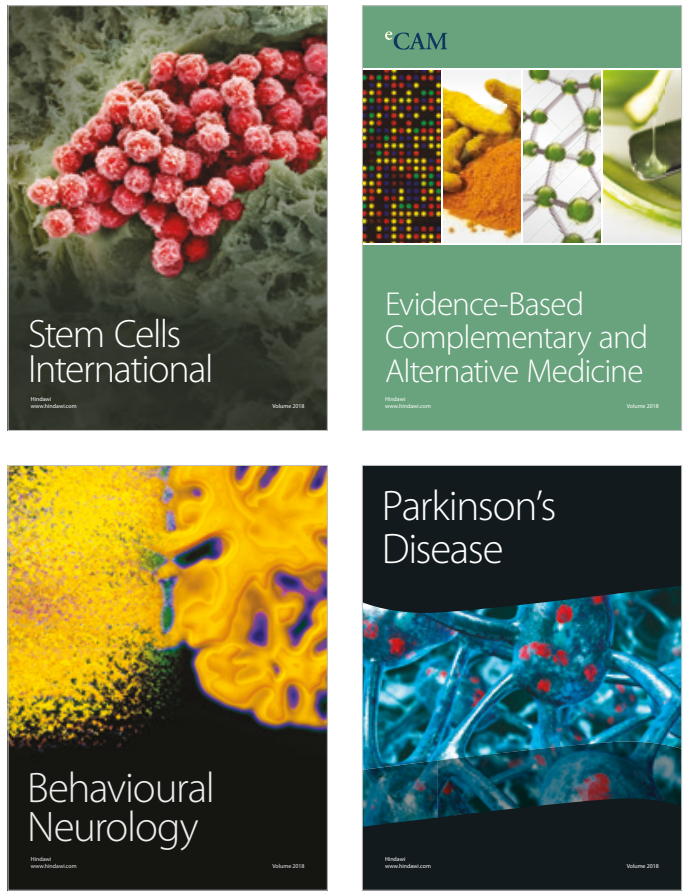

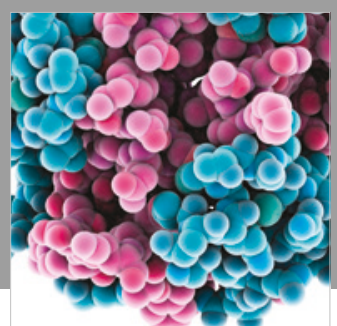

ournal of

Diabetes Research

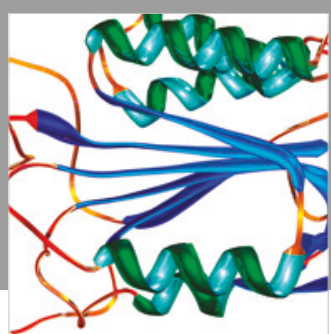

Disease Markers
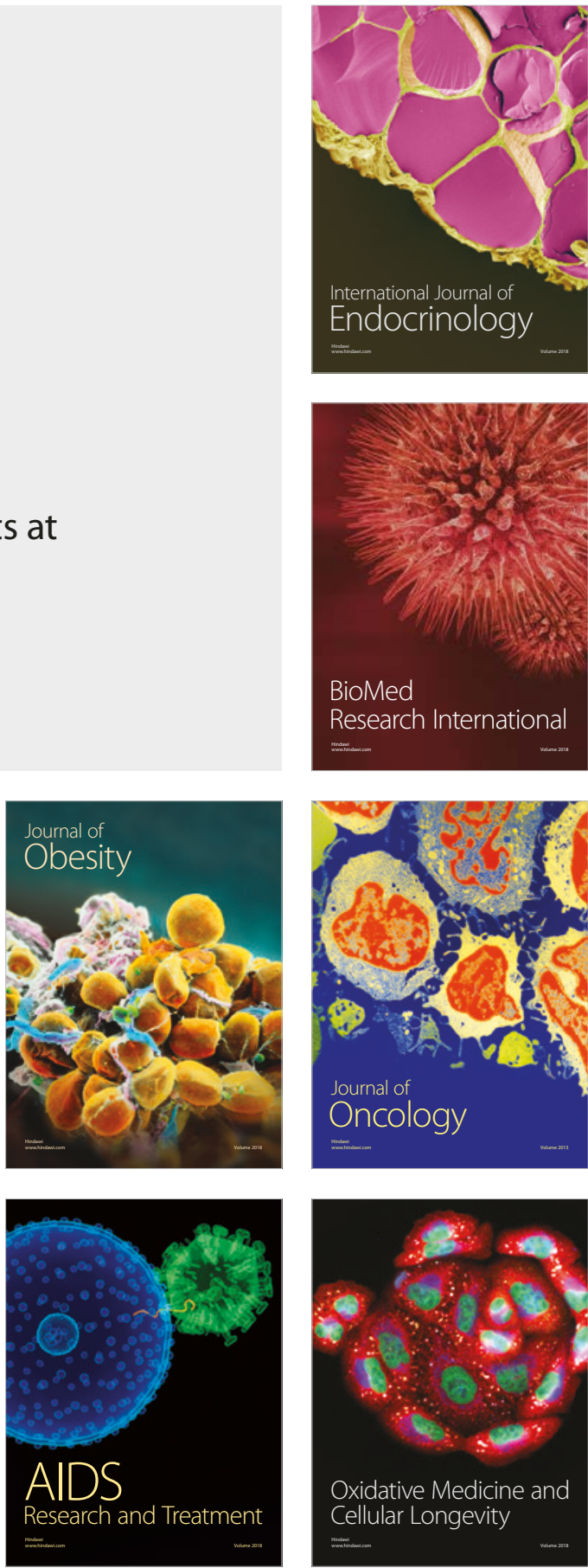